\title{
Atomic Force Microscopy and Magnetic Force Microscopy Study of Model Colloids
}

\author{
M. Raşa, ${ }^{1}$ B. W. M. Kuipers, and A. P. Philipse \\ Van 't Hoff Laboratory for Physical and Colloid Chemistry, Debye Institute, Utrecht University, Padualaan 8, 3584 CH Utrecht, The Netherlands
}

Received August 29, 2001; accepted March 8, 2002; published online May 15, 2002

\begin{abstract}
Atomic force microscopy (AFM) is used to study the size, shape, and polydispersity of a variety of magnetic and nonmagnetic model colloids, previously imaged by transmission electron microscopy (TEM) only. Both height and phase images are analyzed and special attention is given to $3 \mathrm{D}$ morphology and softness of particles, as well as structures and presence of secondary components in the colloid, difficult to investigate with TEM. Several methods of tip characterization followed by deconvolution were applied in order to improve the accuracy of lateral diameter determination. In the case of magnetite particles dispersed in conventional ferrofluids, we explore both experimentally and theoretically the possibility of using magnetic force microscopy (MFM). We propose and discuss several models which allow to estimate the magnetic moment of a single domain superparamagnetic sphere using MFM, which cannot be done with other techniques; alternatively the tip magnetization can be determined. (c) 2002 Elsevier Science (USA)
\end{abstract}

Key Words: colloidal particles; magnetic colloids; atomic and magnetic force microscopy; granulometry.

\section{INTRODUCTION}

Electron microscopy (TEM, SEM) is still the microscopy technique most used for visualizing colloidal particles, though scanning probe microscopy (1) (including among other forms atomic force microscopy (AFM) and magnetic force microscopy (MFM) is now a serious alternative. Scanning probe microscopy relies on the interaction between the sample and a nanometric tip attached to a cantilever, which scans the sample surface in different operating modes. Topographical images of a surface can be taken in contact mode or tapping mode while magnetic domain images can be taken in lift mode. They are briefly described in the following sections.

Previous studies using AFM on colloidal particles imaged mainly gold particles for comparison with other imaging techniques (2) or for applications as tip characterizers and calibrating samples as well as for characterization of coadsorbed

\footnotetext{
${ }^{1}$ To whom correspondence should be addressed. Fax: +(31) 302533870. E-mail: m.rasa@chem.uu.nl.
}

biomolecules (3). Recently, images of silica and polystereneencapsulated silica particles were presented (4) to confirm the synthesis outcome. We imaged in this paper a large variety of colloidal particles (most of them studied with AFM for the first time) not only to confirm some synthesis results but also to make an extensive quantitative analysis of particle shapes and dimensions as well as a qualitative analysis of hardness, clusters, and secondary colloidal components. In comparison with TEM, AFM can give more information by allowing the study of 3D geometry and statistics of particles, by observing features which are transparent for electron microscopy and by imaging soft particles which usually melt in the electron beam of a TEM. AFM also gives detailed shapes of objects after zooming in, at a very high resolution (possibly atomic resolution). For more accurate quantitative determinations several methods of geometrical or numerical tip characterization followed by deconvolution were applied using both a tip characterizer and the samples themselves. The colloids were mainly synthesized in our group, and contain particles having dimensions from a few nanometers to half a micrometer and different shapes and physical properties (bare or encapsulated particles, soft or hard particles, and magnetic or nonmagnetic particles).

For the case of magnetic particles, we also explored MFM. Single-domain superparamagnetic magnetite particles have not been studied before with this technique. Previous studies on MFM were focused mainly on magnetic recording media (5) and multidomain magnetic materials $(6,7)$ as well as on tip characterization (8) and image interpretation $(9,10)$. Closer to our work, there were reported the imaging and remagnetization of Co magnetic dots (with lateral dimensions of $140 \times 250 \mathrm{~nm}$ ) (11) and imaging and magnetic moment estimation of magnetotactic bacteria $(50 \mathrm{~nm}$ in length and $17.5 \mathrm{~nm}$ in radius, if modeled as a cylinder) (12). In this work we investigate both theoretically and experimentally the possibility of magnetic imaging of magnetite nanoparticles (10 $\mathrm{nm}$ in diameter on the average), previously dispersed in cyclohexane and grafted with oleic acid, the dispersion being known as a ferrofluid or magnetic fluid (13). In addition to imaging experiments, the MFM signal was theoretically investigated by calculating the force derivative acting on a model tip due to a single domain superparamagnetic sphere. 


\section{AFM CHARACTERIZATION}

\subsection{Experimental}

Several types of colloidal particles (Table 1) with different properties and dimensions were imaged in air with the Nanoscope IIIa (Multimode AFM, Digital Instruments). The colloids are only briefly decribed here; extensive information can be found in references. Iron particles are synthesized by thermal decomposition of iron pentacarbonyl in a solution of surfactants which adsorb onto the particle surface (14). The magnetite colloids are from a conventional organic ferrofluid as described in (15). The latex spheres are made by emulsion polymerization of fluorinated monomers (16). The spheres are dispersed in water and have as a special feature that their refractive index is close to that of water. The latex spheres are difficult to be imaged with TEM as they easily melt in the electron beam. Teflon colloids are available in aqueous dispersion and have been used to synthesize latex particles with Teflon cores (16). Silica spheres are dispersed in ethanol; their preparation is described in (17). Gibbsite platelets and boehmite needles are obtained from hydrothermal treatment of aluminumalkoxide solutions (18-20). The thickness of these colloids is difficult to measure with TEM. Silica-coated needles, described in (18), are formed by silica polymerization onto boehmite needles.

Mica was used as a substrate, particles being spread on a spinning or fixed but inclined substrate and imaged after drying. AFM measurements were carried out in tapping mode. In this mode the cantilever oscillates close to resonance and the tip only slightly touches the surface. Standard TESP silicon tips (purchased from Digital Instruments) were used.

The freshly cleaved mica has a very small roughness (the measured mean roughness was $R_{a}=0.13 \mathrm{~nm}$ ), favoring the formation of aggregates which appear during drying of the thin layer of colloid, due to the capillary forces. Another experimental problem was the particle attachment to the tip, sometimes observed especially in the case of iron nanoparticles, leading to distorted or more convoluted images. To reduce the aggregation on the substrate, both higher dilutions and substrate derivatization were tried (the last one also helps prevent tip contamination with particles). For the case of negatively charged stabilized particles, a layer of poly- $L$-lysine was first adsorbed on mica. The substrate was immersed in a $0.1 \mathrm{wt} \%$ solution in distilled water for ca. $30 \mathrm{~min}$. The surface was then rinsed in distilled water and dried in air. Then the substrate was immersed in the colloidal dispersion for $15 \mathrm{~min}$, rinsed in the colloid solvent (usually also distilled water), and dried in the air. Formvar coating was tried for the case of surfacted particles. Freshly cleaved mica was immersed in a Formvar solution $(0.25-0.5 \mathrm{~g}$ of Formvar in $100 \mathrm{ml}$ of chloroform) and then spun and dried in air. A drop of colloid was released on the covered substrate which was spun until dry. For spin coating, the sample was attached to a disk rotated by a step-by-step engine at frequencies of tens up to $500 \mathrm{~Hz}$.

\subsection{Results and Discussions}

Magnetic nanoparticles. Iron particles (from sample I) and magnetite particles (from sample M1) are shown in Figs. 1 and 2 respectively. Both height and phase images (Fig. 1) are useful in visualizing the particles. In the phase image (Fig. 1, right image) particles are seen as white cores surrounded by black shells (shell thickness depends on the amplitude setpoint-i.e., on the interaction force between the tip and the sample-as well as on the tip shape). This is because the cantilever phase is shifted differently when the tip touches the particles with its lateral surface (black shell) and with its apex (white core). We mention that this contrast is seen only in the case of solid particles, otherwise tip indentation in particles will lead to a different image. The phase image is thus helpful in seeing 2D cluster composition. Clusters of few particles were observed for all samples, even when highly diluted samples were used.

Magnetite particles were also deposited on the mica substrate in the presence of a magnetic field $(0.1-0.5 \mathrm{~T})$, but no additional cluster formation was observed (Fig. 3a). After increasing the concentration we observed only larger clusters, randomly oriented or of quasispherical shape (Fig. 3b). This

TABLE 1

Samples Imaged with the Atomic Force Microscope

\begin{tabular}{|c|c|c|c|c|c|c|}
\hline Sample & Particles & Stabilization & Solvent & $d_{\mathrm{TEM}}(\mathrm{nm})$ & $d_{\mathrm{SLS}}(\mathrm{nm})$ & $d_{\mathrm{AGM}}(\mathrm{nm})$ \\
\hline M1 & Magnetite & Oleic acid & Cyclohexane & 8.6 & - & 7 \\
\hline M2 & Magnetite & Oleic acid & Cyclohexane & 9.1 & - & - \\
\hline I & Iron & Oleic acid & Cyclohexane & 9.5 & - & - \\
\hline $\mathrm{L}$ & Latex & Charge (negative) & Distilled water & - & 188 & - \\
\hline $\mathrm{TL}$ & Teflon core & Negative charged latex & Distilled water & - & - & - \\
\hline $\mathrm{T}$ & Teflon & Charge (negative) & Distilled water & - & - & - \\
\hline $\mathrm{S}$ & Silica & Charge (negative) & Ethanol & - & 120 & - \\
\hline $\mathrm{G}$ & Gibbsite & Polyisobutylen & Toluene & 232 & - & - \\
\hline BS & Boehmite core & Negative charged silica & Ethanol & - & - & - \\
\hline
\end{tabular}

Note. $d_{\mathrm{TEM}}$ is the mean diameter measured with TEM, $d_{\mathrm{SLS}}$ is the mean diameter measured using static light scattering and $d_{\mathrm{AGM}}$ is the mean "magnetic diameter" measured with the alternating gradient magnetometer. 


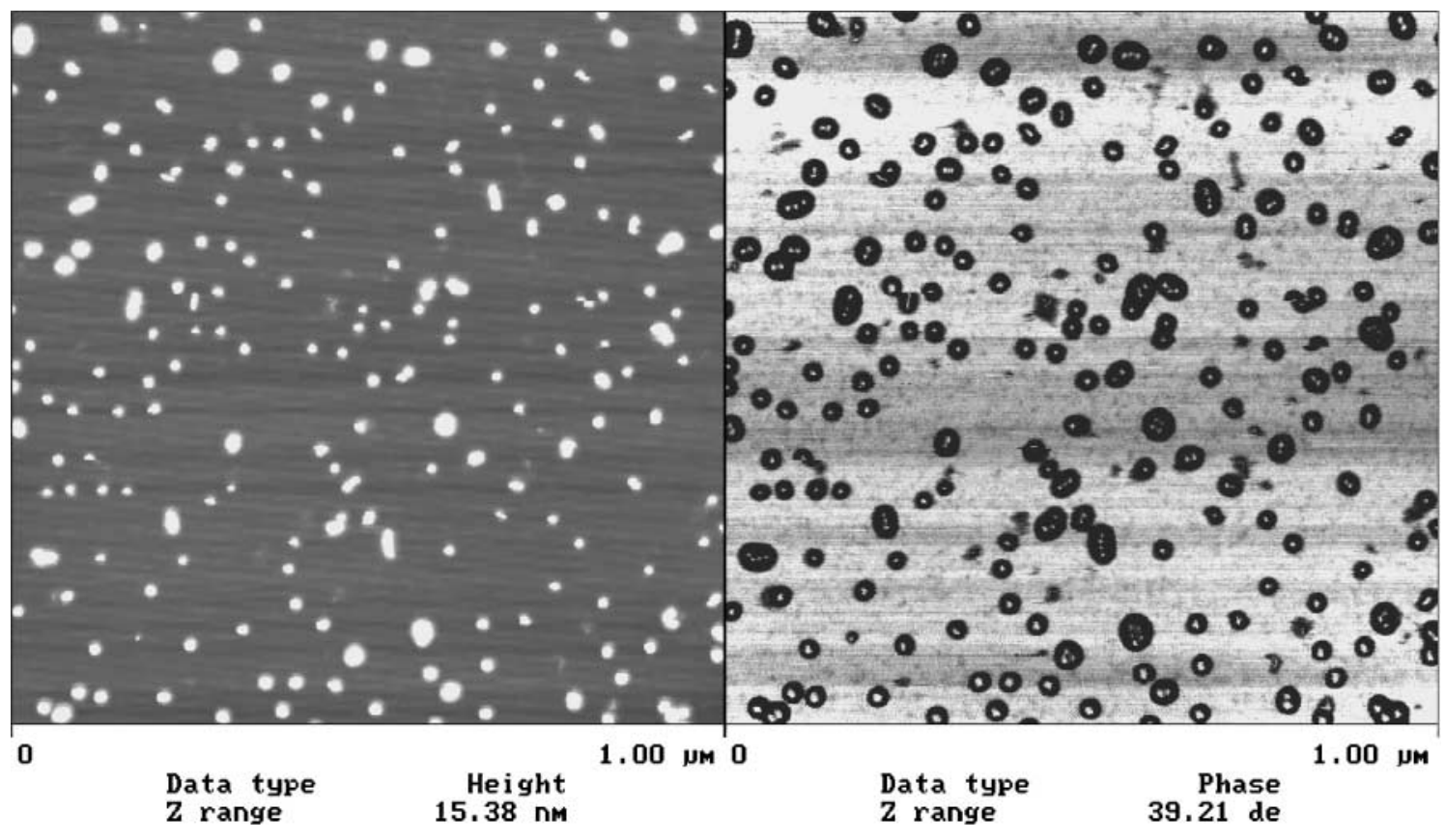

FIG. 1. Height and phase image of iron particles.

is explained by the fact that the capillary forces are stronger than the dipolar forces which act between magnetic particles. The sticky polymer coating (Formvar) did not significantly reduce the effect of capillary forces (particles from a very dilute sample, deposited on a Formvar layer, are presented in Fig. 4). Consequently, we think that most of the aggregates form during drying, but preformed aggregates from the colloid, maintained by the van der Waals attraction, are also possible.

Height and (lateral) diameter distributions of particles were analyzed using height images. Obvious aggregates, as seen in the phase images, were manually removed. The results for both types of particles are presented in Table 2. In comparison with the TEM mean diameter, $d_{\text {TEM }}$, the particle mean height, $h$, obtained with AFM is slightly smaller, mainly due to the deviation of particles from spherical shape. The particle indentation in the substrate due to the tip-particle interaction can be avoided by reducing the tip-sample interaction force (i.e., by increasing the

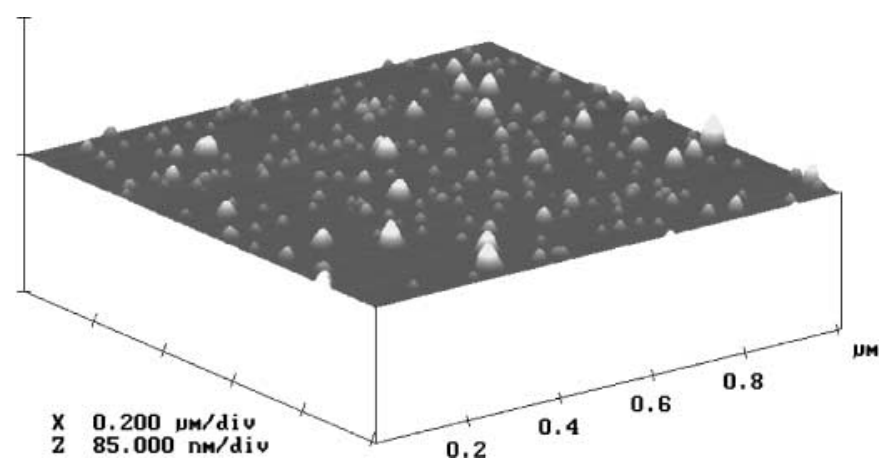

FIG. 2. Surface view of magnetite particles. amplitude setpoint). The AFM mean diameter, $d$, also further referred to as the lateral diameter or measured diameter from the image, is larger than the TEM mean diameter due to the tip convolution. The AFM mean diameter determination can be affected by the arbitrary height of the chosen analysis plane (also called the threshold plane), which is a plane above and parallel to the substrate, raised in order to eliminate noise and to define the particle boundaries. Therefore the substrate was flattened (after the particles in the image were masked for this operation) and a median filter was then applied once in order to reduce noise, so that we reduced the threshold height as much as possible.

We mention that the mean TEM diameter is in good agreement with the mean magnetic diameter of magnetite particles (M1), measured with an alternating gradient magnetometer and determined as in (21), because of a nonmagnetic layer approximately $0.86 \mathrm{~nm}$ thick.

The accuracy of the AFM mean diameter determination can be improved by deconvolution. In the case of such small particles, only the tip apex determines the convolution. If both the tip apex and the particle are considered spherical, a simple geometrical

TABLE 2

Statistics on AFM Imaged Magnetic Nanoparticles

\begin{tabular}{lccc}
\hline \multicolumn{1}{c}{ Particles } & $h(\mathrm{~nm})$ & $d(\mathrm{~nm})$ & $p(\%)$ \\
\hline Magnetite (M1) & 6.7 & 16.6 & 15.1 \\
Iron (I) & 7.7 & 15.5 & 12.2 \\
\hline
\end{tabular}

Note. Mean height $(h)$, mean diameter $(d)$, and diameter polydispersity $(p)$ were determined. 


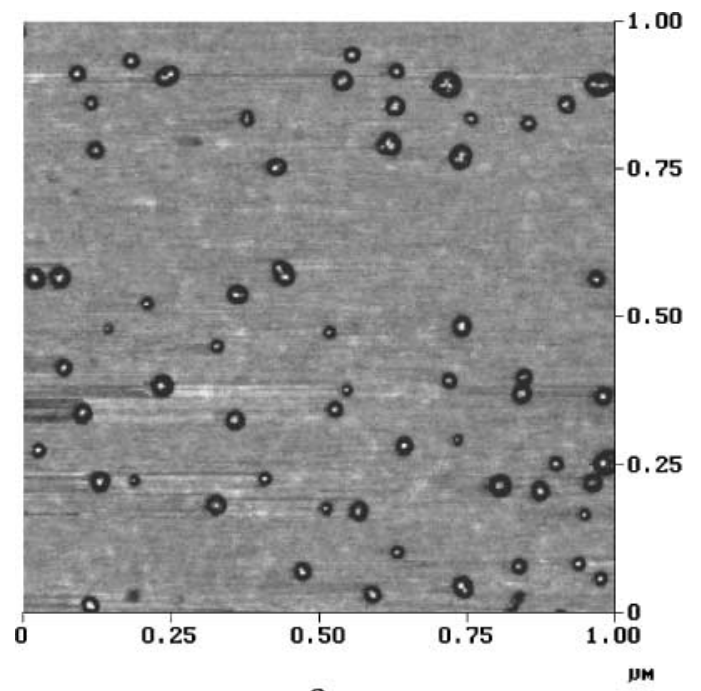

a.

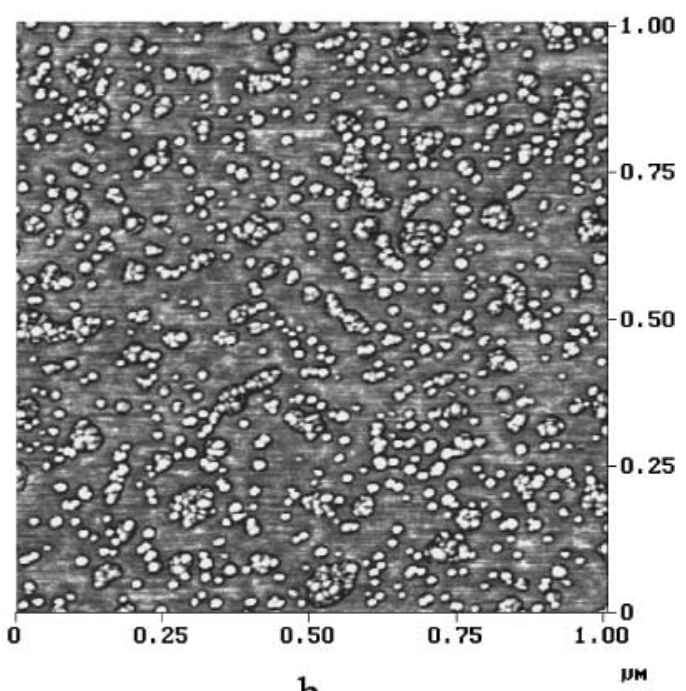

b.

FIG. 3. Phase image of magnetite particles deposited on mica in the presence of an external magnetic field, from a very dilute colloid (a) and from a somewhat more concentrated one (b).

exercise shows that (3)

$$
r_{c}=2 \sqrt{r R}
$$

where $r$ is the particle radius, $R$ is the tip radius, and $r_{c}$ is the AFM radius of a particle, as seen in the image. Taking into account an average nominal tip radius of $7.5 \mathrm{~nm}$, a particle with $r=2 \mathrm{~nm}$ is seen almost four times larger while a particle with $r=8 \mathrm{~nm}$ is seen almost two times larger. However, the tip radius can vary considerably from one probe to another, so that in order to perform deconvolution, tip characterization is necessary. For a certain tip, the apex radius was estimated by means of three methods: "blind tip characterization" (22) (method I in this paper) applied on an image of magnetite particles (sample M2, mean diameter of $19 \mathrm{~nm}$ in the image - not presented), the same method applied on an image of a "Nioprobe" tip characterizer with very sharp peaks (II), and finally, a manual estimation technique using the same "Nioprobe" image (III)

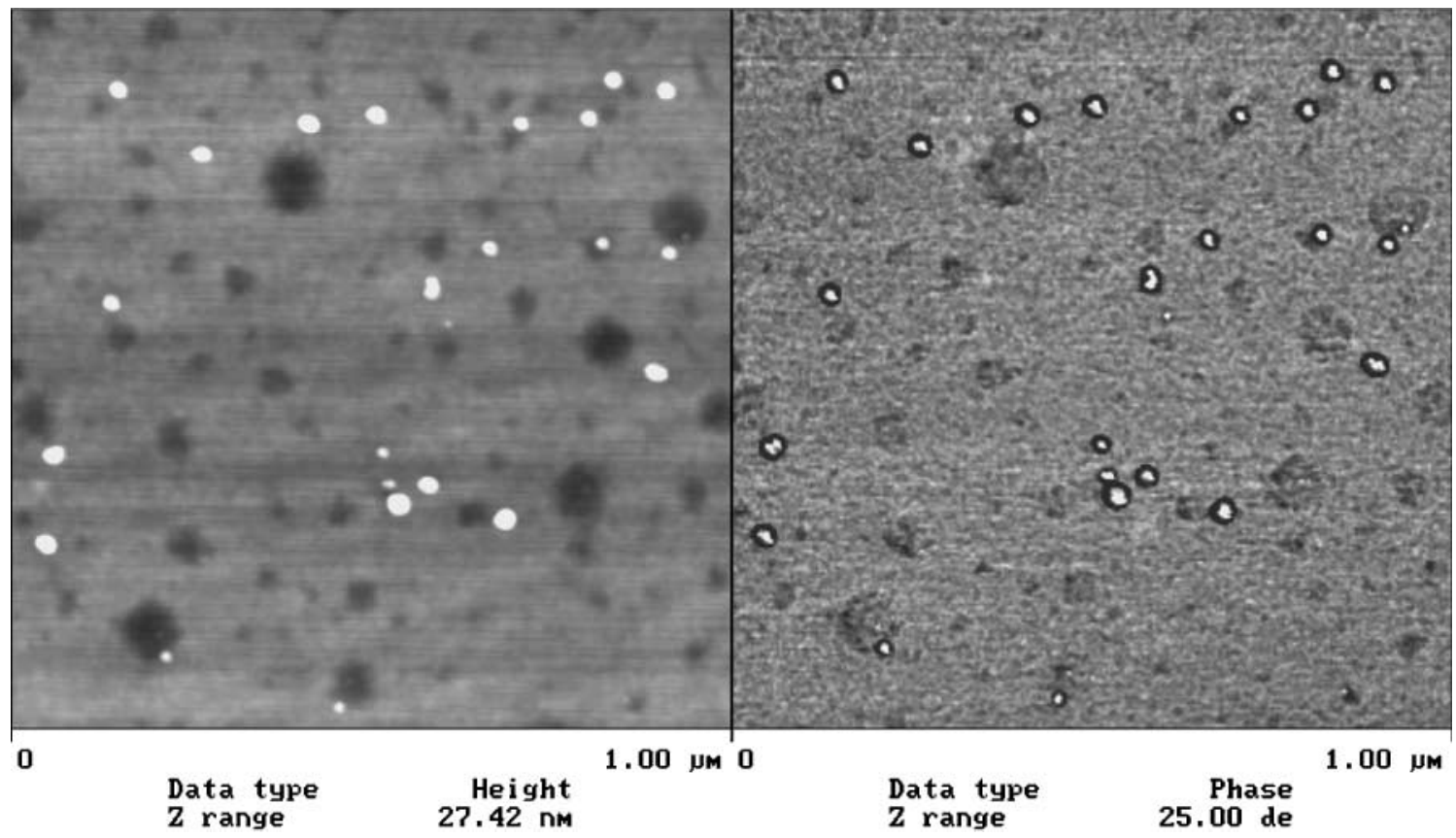

FIG. 4. Magnetite particles on a Formvar-coated mica substrate. 
TABLE 3

Results of Tip Characterization and Deconvolution for Magnetite Particles (M2) with a Measured Mean Diameter of $d=19.2 \mathrm{~nm}$ and Mean Height of $h=6.5 \mathrm{~nm}$ in the Original Image

\begin{tabular}{lcccc}
\hline Method & $R_{1}(\mathrm{~nm})$ & $R_{2}(\mathrm{~nm})$ & $d^{\prime}(\mathrm{nm})$ & $h^{\prime}(\mathrm{nm})$ \\
\hline I & 6.8 & 4.8 & 15.4 & 6.1 \\
II & 9.5 & 6.0 & 14.3 & 2.4 \\
III & $18.3 \pm 5$ & $18.3 \pm 5$ & 15.2 & 2.5 \\
\hline
\end{tabular}

Note. $R_{1,2}$ are the tip radiuses, $d^{\prime}$ the corrected mean diameter, and $h^{\prime}$ the corrected mean height.

(23). In the last case the mean radius of the tip was estimated from the highest peaks in the image after measurement of their height $(H)$ and half width $(W): R=\left(W^{2}+H^{2}\right) /(2 H)(23)$. In the other cases we used "Deconvo 1.1" software, which fitted the surface of the tip with an ellipsoid so that we obtained two radii $R_{1}$ and $R_{2}$. The determined radii are presented in Table 3 and a significant difference is observed between the manual and numerical estimations. In contrast, the corrected lateral diameters, obtained after an erosion procedure was applied by using Deconvo 1.1, were close to each other (Table 3). We also observed that the erosion procedure affects significantly the height of particles in the case of methods II and III, which normally should not be affected by such a procedure. Therefore we consider the first method a better one. After removing the aggregates, we obtained a mean corrected diameter of $12 \mathrm{~nm}$, in better agreement with the TEM mean diameter $(9.1 \mathrm{~nm})$. We mention finally that, after applying Eq. [1], in which the nominal average tip ra-
TABLE 4

Statistics on AFM Imaged Nonmagnetic Particles

\begin{tabular}{lcccccc}
\hline \multicolumn{1}{c}{ Particle } & $d(\mathrm{~nm})$ & $h(\mathrm{~nm})$ & $d / h$ & $l(\mathrm{~nm})$ & $w(\mathrm{~nm})$ & $l / w$ \\
\hline Latex (L) & 728 & 88.0 & 8.3 & 767 & 710 & 1.08 \\
Teflon (TL) & 387 & 168 & 2.3 & 406 & 372 & 1.09 \\
Teflon (T) & 217 & 163 & 1.3 & 238 & 204 & 1.16 \\
Silica (S) & 145 & 112 & 1.3 & 161 & 136 & 1.18 \\
Gibbsite (G) & 213 & 12.4 & 17.2 & 245 & 178 & 1.37 \\
Boehmite (BS) & - & 24.1 & - & 228 & 30.7 & 7.43 \\
\hline
\end{tabular}

dius is used $(7.5 \mathrm{~nm})$, one obtaines a real radius of $r=12.4 \mathrm{~nm}$ if $r_{c}$ is $19.3 \mathrm{~nm}$.

Nonmagnetic particles. Latex particles (L) are presented in Fig. 5. The specimen was prepared on freshly cleaved mica. Particles are seen as black spots in the phase image (due to tip indentation), suggesting that they are soft particles. Granulometry performed using the height image showed a mean lateral diameter, $d, 8.3$ times larger than the mean height, $h$, of the particles (Table 4). This is a consequence of the flattening of the soft particles on the mica substrate and of tip indentation. It also explains why the lateral diameter is much larger than the diameter of particles obtained from static light scattering, $d_{\mathrm{SLS}}$. One can also observe in this figure very small particles (a good contrast is given by the phase image) which probably are due to residual monomer in the latex dispersions. We can also infer that the latex particles are very close to the spherical shape in the dispersion, because the circular symmetry is preserved in any plane parallel to the substrate: in Table 4 the ratio between the

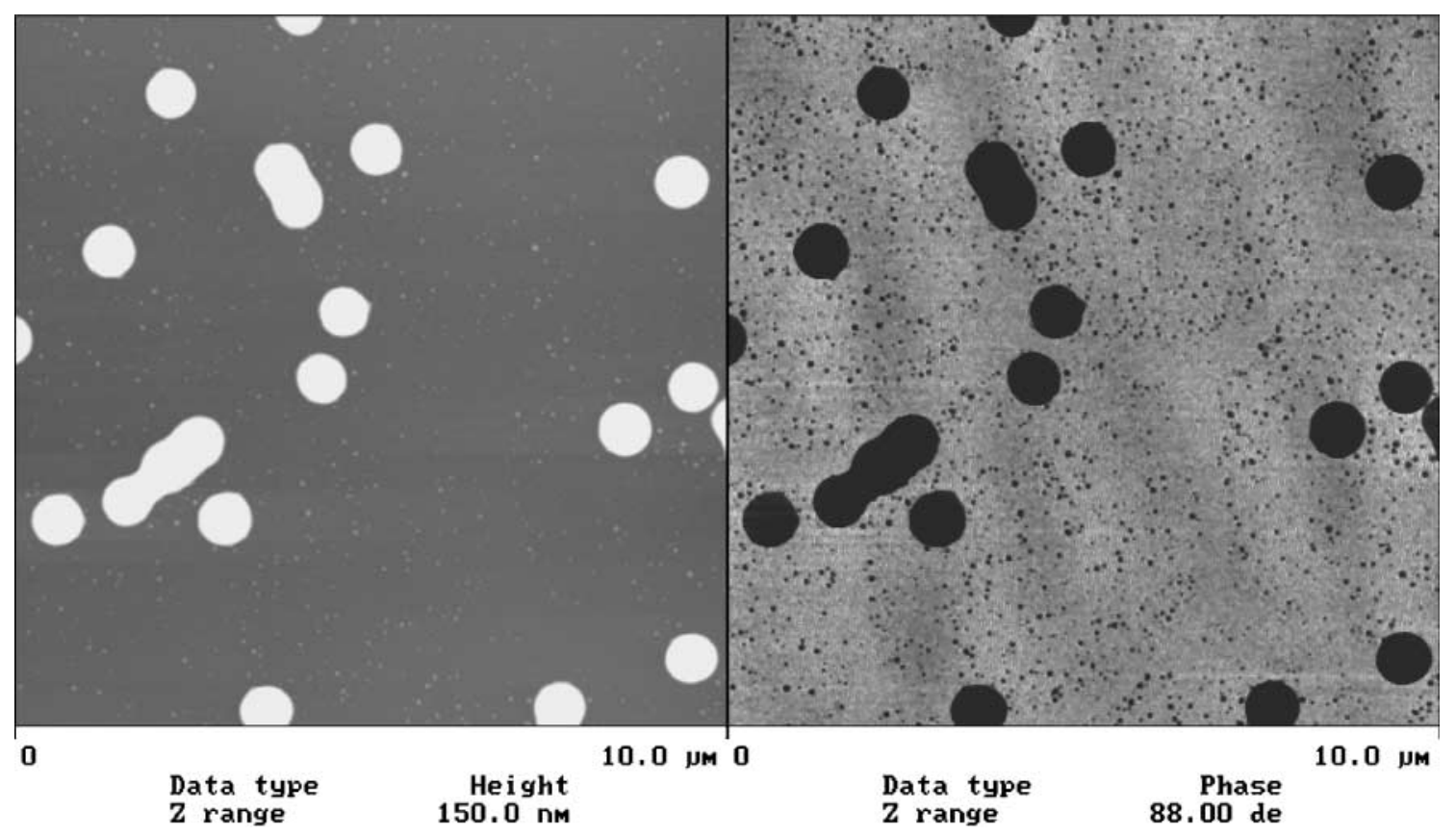

FIG. 5. Height and phase image of latex particles on mica. 


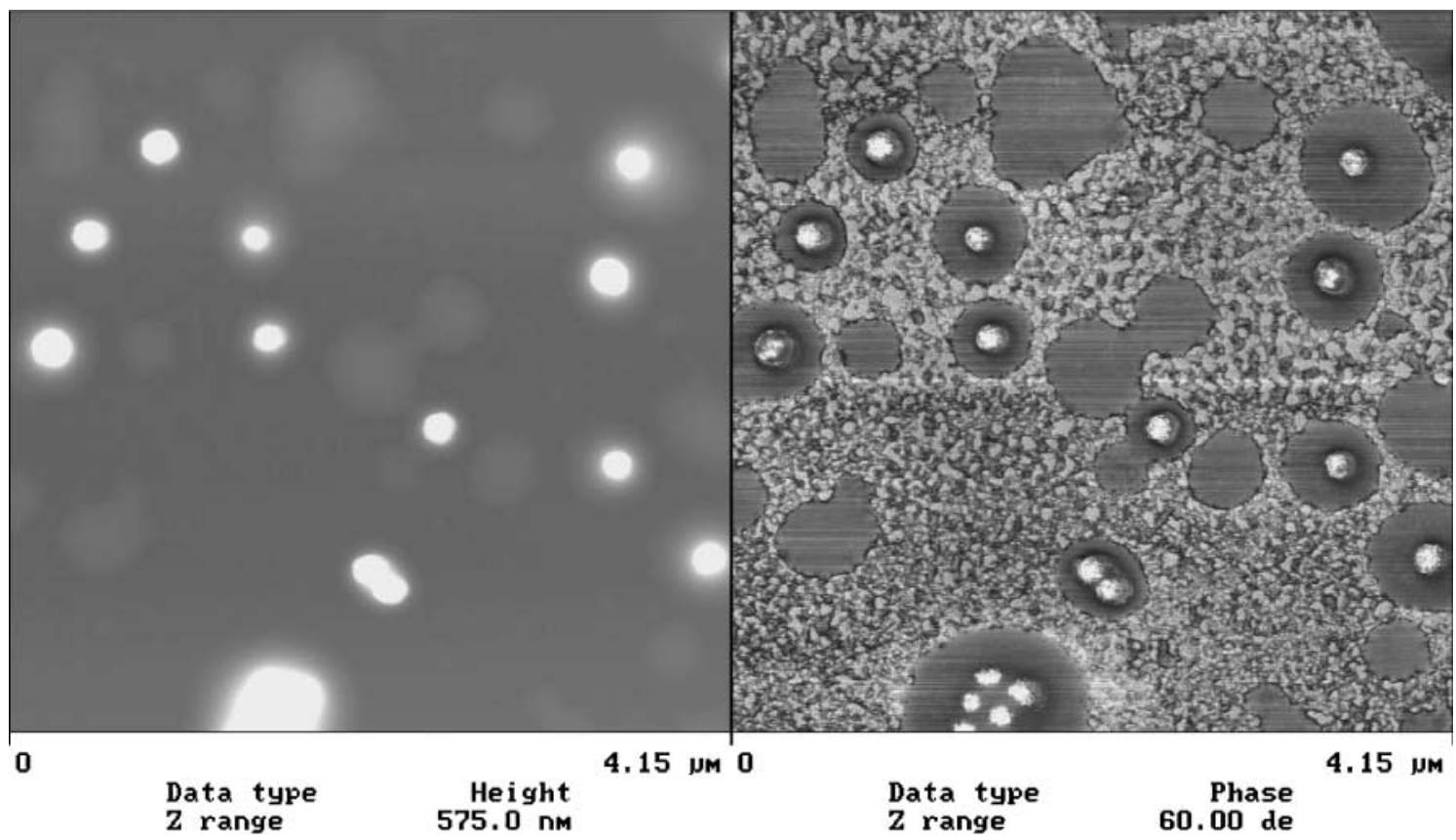

FIG. 6. Teflon core particles coated with latex on a poly- $L$-lysine coated mica.

mean largest lateral size (also called mean length), $l$, and the mean smallest lateral size (also called mean width), $w$, of these particles is very close to one. Electron microscopy of sample $\mathrm{L}$ was very difficult, because of particle melting, while the small particles were not observed at all.

Teflon particles covered with latex (TL) could not be imaged on mica, because, regardless of how diluted the samples were, aggregates formed. Single particles (together with some small clusters) were seen only on mica substrates covered with poly$L$-lysine (Fig. 6). The ratio between the lateral diameter and height is much smaller than in the case of sample L (Table 4), showing in fact a relatively hard Teflon core, as it also results from the phase image contrast, and flattened latex shell with variable thickness. Latex shells are very clearly seen both in the height and phase image, the last one providing also a nice image of cluster composition in a good contrast. Some flat latex spots are also observed, unlike in TEM pictures which were doubtful due to the particle deformation because of heating by the electron beam. Sample T contains also teflon particles but without latex shell. In Fig. 7a, single particles (together with

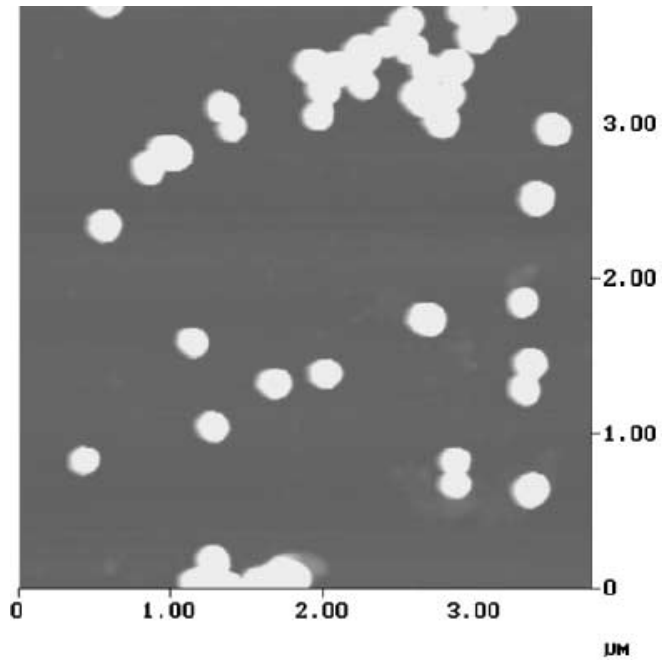

a.

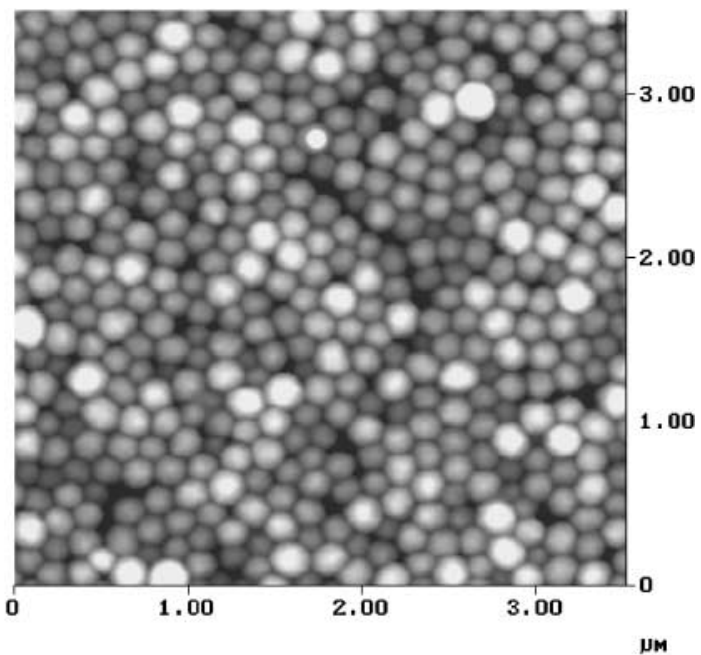

b.

FIG. 7. Teflon particles on a poly- $L$-lysine coated mica: height images containing several individual particles (a) and structured particles (b). 


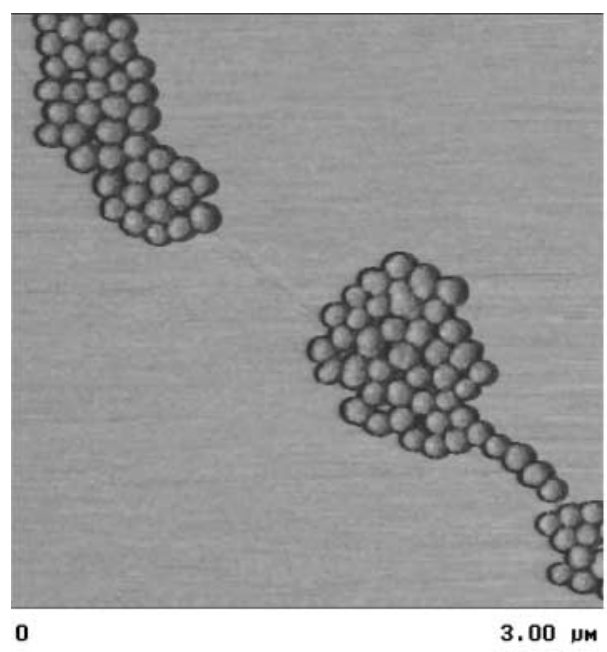

a.

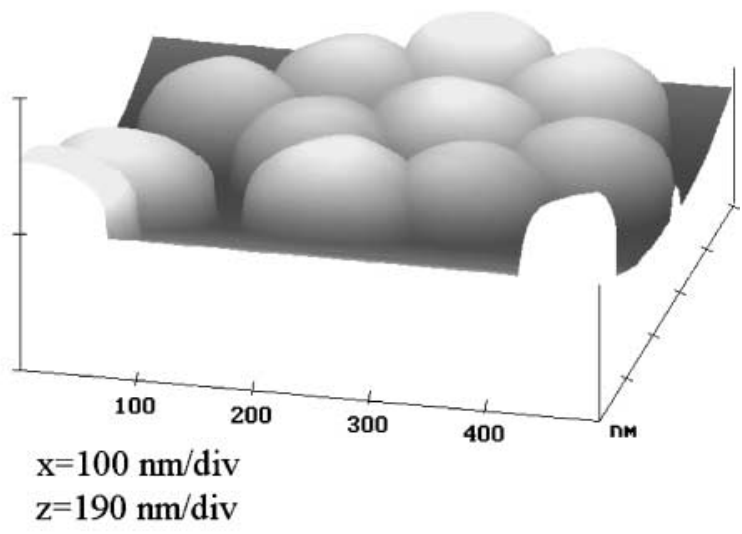

b.

FIG. 8. Phase image of silica particles on mica (a) and a height image surface view of a zoomed cluster (b).

some aggregates) were observed only after poly- $L$-lysine coating. It is worth mentioning that this sample is very clean, no other additional features are present in the image. In comparison with TL samples, $d / h$ ratio is smaller because only hard teflon particles were measured (Table 4). Sometimes particle condensation on the derivatized substrate was observed (Fig. 7b).

In Fig. 8 silica particles (sample $S$ ) were imaged before substrate derivatization, and in Fig. 9 after poly- $L$-lysine coating of the substrate. In the left image of Fig. 8, silica particles are everywhere aggregated, some of clusters having the hexagonal packing structure, as in the case of colloidal crystals. The same phase image shows hard particles (white cores surrounded by the black shells). After poly- $L$-lysine coating, we were able to see and measure individual particles. Even though silica are hard

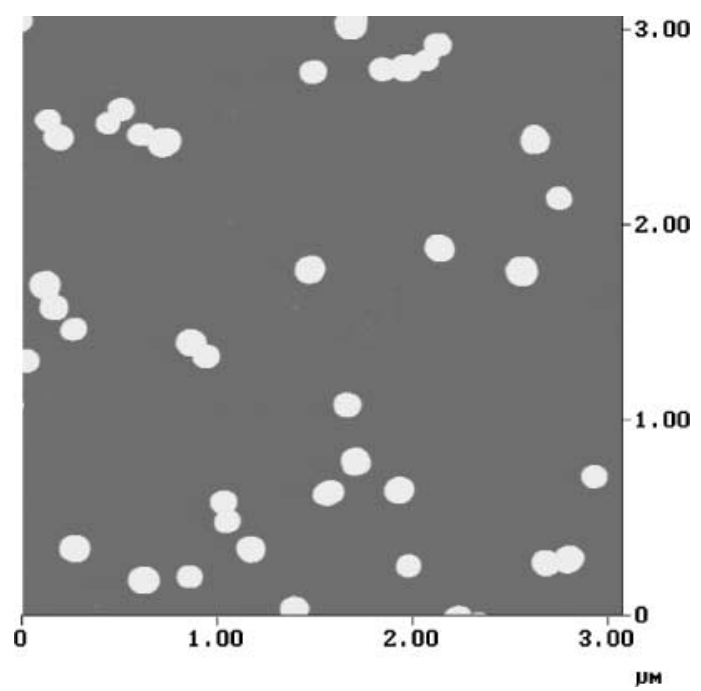

FIG. 9. Silica particles on a poly- $L$-lysine coated substrate-height image. particles, a somewhat larger diameter was measured than height (Table 4). This is due to the tip convolution, which is now determined by the mean half angle $\theta_{0}$ of the tip instead of the apex radius $R$ because of the larger dimensions of particles. From Fig. 10 we obtained

$$
\begin{aligned}
& r_{c}= \\
& r\left(\cos \theta_{0}+\sqrt{\cos ^{2} \theta_{0}+\left(1+\sin \theta_{0}\right)\left(-1+\left(\tan \theta_{0} / \cos \theta_{0}\right)+\tan ^{2} \theta_{0}\right)}\right) .
\end{aligned}
$$

For a particle (real) diameter of $112 \mathrm{~nm}$ (corresponding to the real radius $r$ in Eq. [2]), a value of $150 \mathrm{~nm}$ is obtained for particle lateral diameter (corresponding to the radius $r_{c}$ in Eq. [2]). A mean value of $17^{\circ}$ was considered for the tip half angle in Eq. [2].

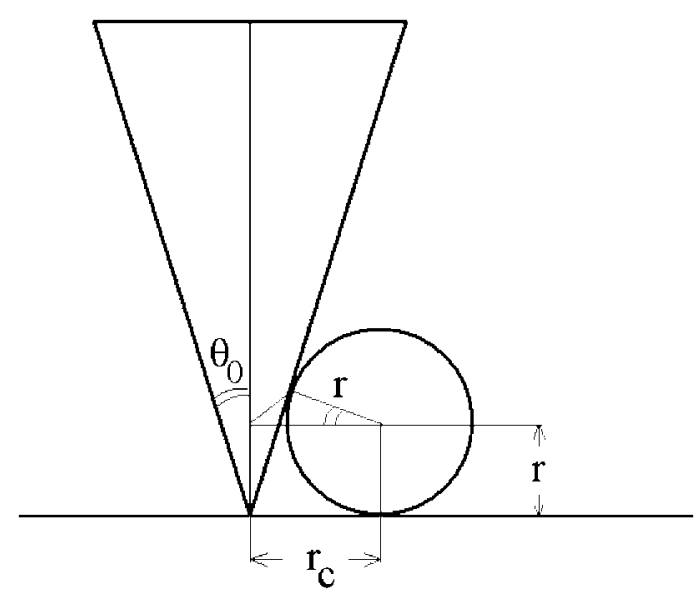

FIG. 10. Apparent radius $r_{c}$ of a particle due to the tip convolution in the case of a particle radius much larger in comparison with the tip apex radius. 


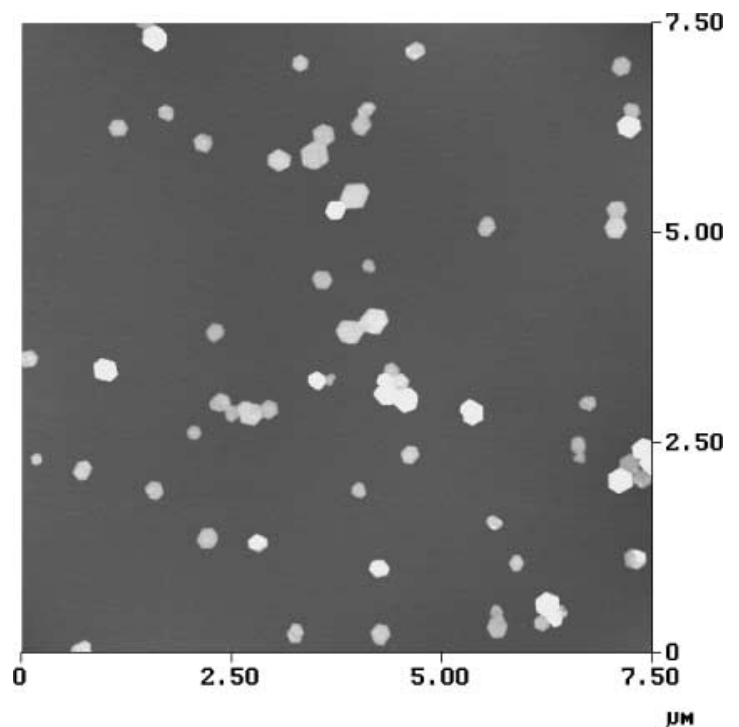

FIG. 11. Height image of gibbsite platelets.

This result, in very good agreement with the determinations presented in Table 4, shows that, indeed, the measured mean lateral diameter $d=145 \mathrm{~nm}$ is larger than the real mean diameter (approximately equal to the mean height $h=112 \mathrm{~nm}$ ) because of the tip convolution. We can also conclude from such an analysis that silica particles can be indeed considered as undeformable spheres.

Gibbsite platelets are presented in Fig. 11. In this case the height of particles is of order of $10 \mathrm{~nm}$ while the width is of order of $200 \mathrm{~nm}$ (the averaged values from several pictures are presented in Table 4). The convolution is negligible in this case and the diameter is not affected practically by the choice of the threshold plane because the section area, perpendicular to the vertical direction, is constant. Both $d / h$ and $l / w$ ratios are reliable values in this case. If the synthesis procedure can provide monodisperse platelets, they can be used for the XYZ calibration of the AFM scanner. The TEM "diameter," which was measured as the average distance between the opposed vortices of the hexagonal particles, lies between the AFM mean diameter $d$ (which assigned the measured area to the area of a circular disk) and mean length $l$, as it normally should be.

Finally, boehmite rods covered with silica were analyzed (Fig. 12). A poly- $L$-lysine coated mica was used. From Fig. (a) we determined the mean height $h$ as well as the mean aspect ratio of particles (Table 4). A zoomed measurement shows (Fig. 12b) a granular contour of the silica shell and small spheres, which are not seen normally in a TEM picture. As in the case of sample $\mathrm{G}$, the convolution for rods is negligible. The small spheres, which lie on the substrate, can be easily mistaken for small silica particles but they were not observed when the rods were measured on an uncoated freshly cleaved mica. The small spheres are poly- $L$-lysine peaks. Their presence can be reduced if a more dilute poly- $L$-lysine solution is used.

\section{MFM STUDY}

\subsection{Experimental}

MFM experiments on single-domain superparamagnetic magnetite $\left(\mathrm{Fe}_{3} \mathrm{O}_{4}\right)$ particles were performed in dynamic lift mode, in air, after the topography was measured in tapping mode. In dynamic lift mode the cantilever oscillates near resonance at a certain distance (called lift height) from the sample, while the tip follows the topographic profile. Phase detection and frequency modulation were used for obtaining the magnetic images.

Electrical forces and particle attachment to the tip were suppressed by depositing a gold layer of at least $5 \mathrm{~nm}$ on the sample

b.

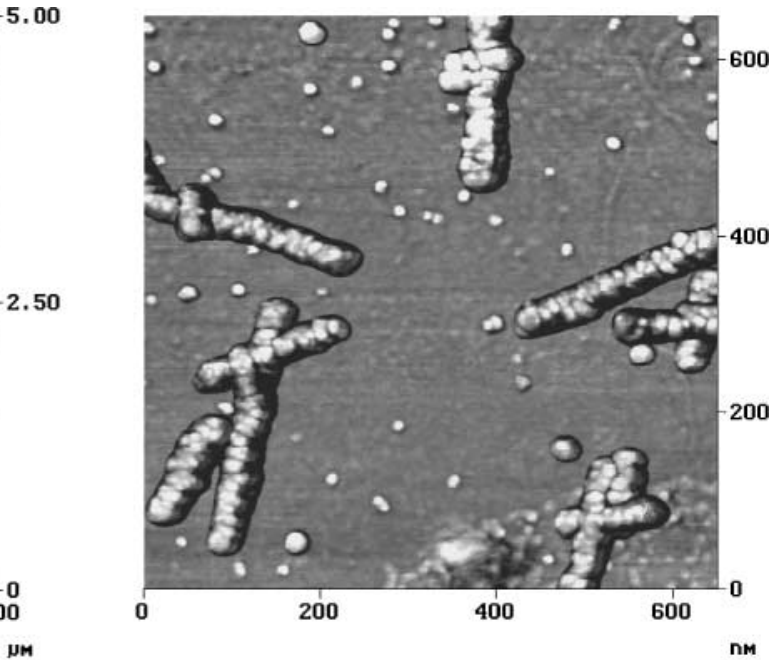

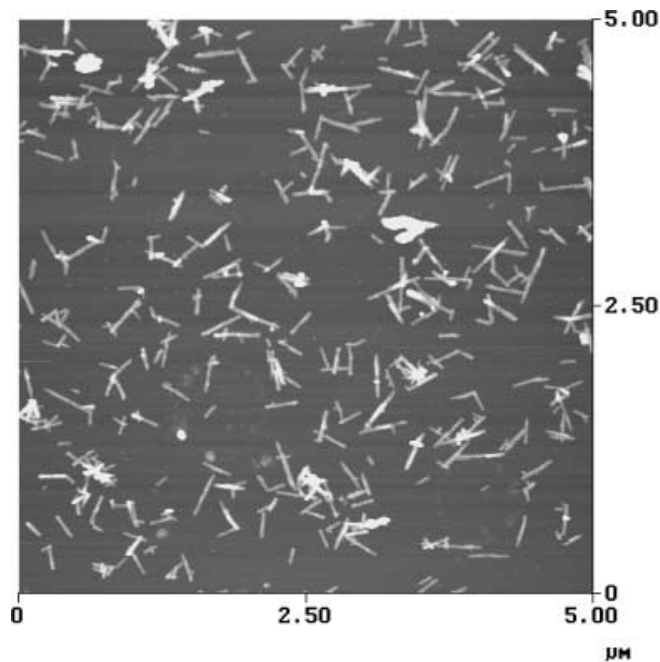

a.

FIG. 12. Height image of boehmite core rodlike particles (a) and phase image of the same particles recorded for details of rod silica contour (b). 
surface using a sputter coater. We used standard magnetic tips (MESP tips, purchased from Digital Instruments) with a 50-nm $\mathrm{CoCr}$ coating. The physical characteristics of a tip were determined from an SEM image and the magnetic hysteresis was measured with an alternating gradient magnetometer. High-moment magnetic tips (MESP-HM, Digital Instruments) were also used.

\subsection{Results and Discussion}

In dynamic lift mode, the vibrating cantilever is sensitive to the derivative of the magnetic force acting on the tip $\left(F^{\prime}=\partial F_{z} / \partial z\right)$, which changes the resonant frequency $\left(v_{0}\right)$ of the free oscillating probe, according to the relation (1)

$$
\Delta v \approx-v_{0} F^{\prime} /(2 k)
$$

where $k$ is the spring constant of the cantilever. Equation [3] is valid for small oscillation amplitudes of the cantilever, for a constant air damping of the cantilever oscillations, and for $F^{\prime} \ll k$ (the last always valid in our experiments of weak tipsample interaction). It is also assumed in this paper that $\mathbf{n} \approx \mathbf{z}$ where $\mathbf{n}$ is the unit vector normal to the cantilever plane and $\mathbf{z}$ the unit vector of the vertical direction; i.e., the cantilever is near parallel to the sample and the oscillation amplitude is very small.

Experimentally, a topographic interference ( $\Delta v$ up to $4-5 \mathrm{~Hz}$ ) at lift heights varied between 15 and $45 \mathrm{~nm}$ was noticed many times in the lift mode image in which the particles look almost the same as in topographical image (Fig. 13). Because this fake (i.e., nonmagnetic) signal is weaker but still measurable up to $45 \mathrm{~nm}$, it cannot be assigned to the van der Waals interaction. Moreover, both magnetic interaction with superparamagnetic particles and van der Waals interaction, which are attractive, would lead to a negative contrast (particles should be seen black), unlike in Fig. 13. These make it very difficult to identify the magnetic signal. Sometimes no signal was measured at all.

In order to see if such small magnetic particles are measurable with MFM, we calculated the shift in the resonant frequency in several ways. From the SEM image, the height of the tip $L=$ $14.5 \mu \mathrm{m}$ and the apex radius $R=51.4 \mathrm{~nm}$ were determined. The measured spring constant was $k=2.4 \mathrm{~N} / \mathrm{m}$. The magnetization of the CoCr alloy thin film $M_{t}=7.2 \times 10^{5} \mathrm{~A} / \mathrm{m}$, was taken from (12) and it is close to the value obtained in (24). In general, the force acting on the tip can be obtained by integrating the tip-sample force density,

$$
F=\mu_{0} \nabla\left(\mathbf{M}_{\mathbf{t}} \cdot \mathbf{H}_{\mathbf{s}}\right),
$$

over the tip volume. $\mu_{0}$ is the vacuum permeability and $H_{s}$ the stray field of the sample.

First, a point dipole-dipole interaction between the apex of the tip, considered a sphere with the radius $R / 2$, and a spherical particle was taken into account. The mean magnetic moment of the particle was obtained from the magnetometric measurements of a diluted magnetic fluid sample. After magnetogranulometric analysis done as in (21), we found $m_{p}=8 \times 10^{-20} \mathrm{Am}^{2}$. The magnetic moment of the particle is assumed parallel to the tip magnetization (see the discussion in the eighth paragraph). The tip apex magnetic moment was estimated to be

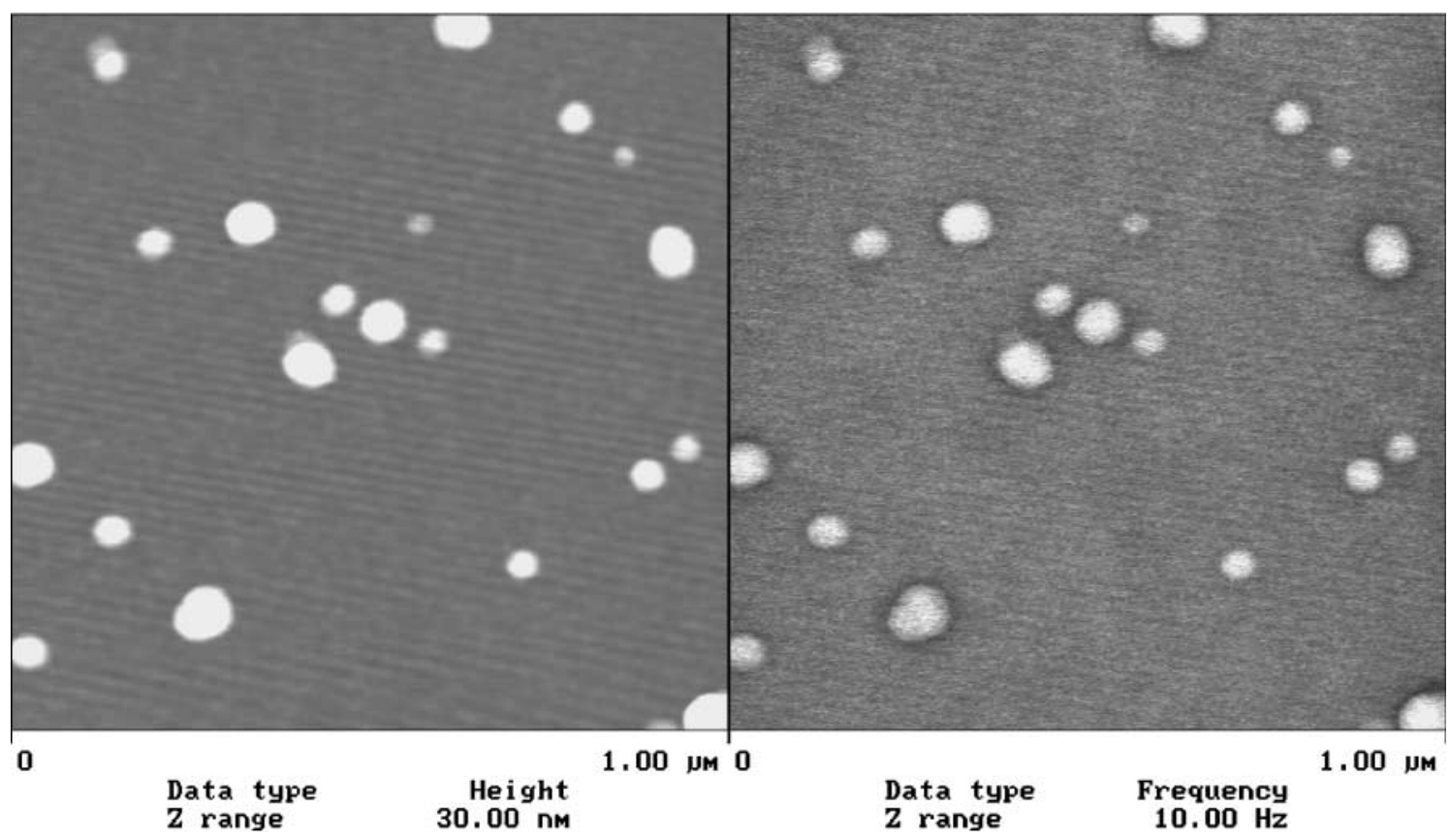

FIG. 13. Topographical image of magnetite particles (left) and fake magnetic signal recorded in lift mode(right). The sample surface was covered with a 5.5-nm gold layer. 
$m_{t}=5.1 \times 10^{-17} \mathrm{Am}^{2}$. Taking into account the dipolar field expression,

$$
\mathbf{H}_{\mathbf{s}}=\frac{1}{4 \pi}\left[\frac{3\left(\mathbf{m}_{\mathbf{p}} \cdot \mathbf{r}\right)}{r^{5}} \mathbf{r}-\frac{\mathbf{m}_{\mathbf{p}}}{r^{3}}\right],
$$

the force derivative in this case will be

$$
F^{\prime}=\frac{6 \mu_{0} m_{p} m_{t}}{\pi\left(s+z_{0}\right)^{5}}
$$

where $s$ is the distance between the surfaces of the tip apex and particle and $z_{0}=R / 2+d_{m} / 2+\delta . d_{m}$ is the mean magnetic diameter (Table 1 ) and $\delta$ is the thickness of the nonmagnetic layer, determined from magnetization and TEM measurements (21) $(\delta=0.8 \mathrm{~nm})$.

The force derivative was then calculated, for the case of a uniform tip magnetization, starting from

$$
F^{\prime}=\mu_{0} \mathbf{M}_{\mathbf{t}} \cdot\left(\frac{\partial^{2}}{\partial z^{2}} \int_{V} \mathbf{H}_{\mathbf{s}}(\mathbf{r}) \mathrm{d} \mathbf{r}\right) .
$$

$V$ is the volume of the magnetic coating of the tip and $\mathbf{H}_{\mathbf{s}}$ the dipolar field produced by the magnetite particle considered spherical $\left(d_{m}=7 \mathrm{~nm}\right)$, outside the particle, given by Eq. [5]. The tip is considered a cone with a magnetic coating of $50 \mathrm{~nm}$ and with a mean half angle of $\theta_{0}=17^{\circ}$. We assume that the tip is uniformly magnetized in the $-\mathrm{O} z$ direction, it is positioned above the particle center, and $\mathbf{m}_{\mathbf{p}} \| \mathbf{M}_{\mathbf{t}}$ (Fig. 14). In this case the maxi-

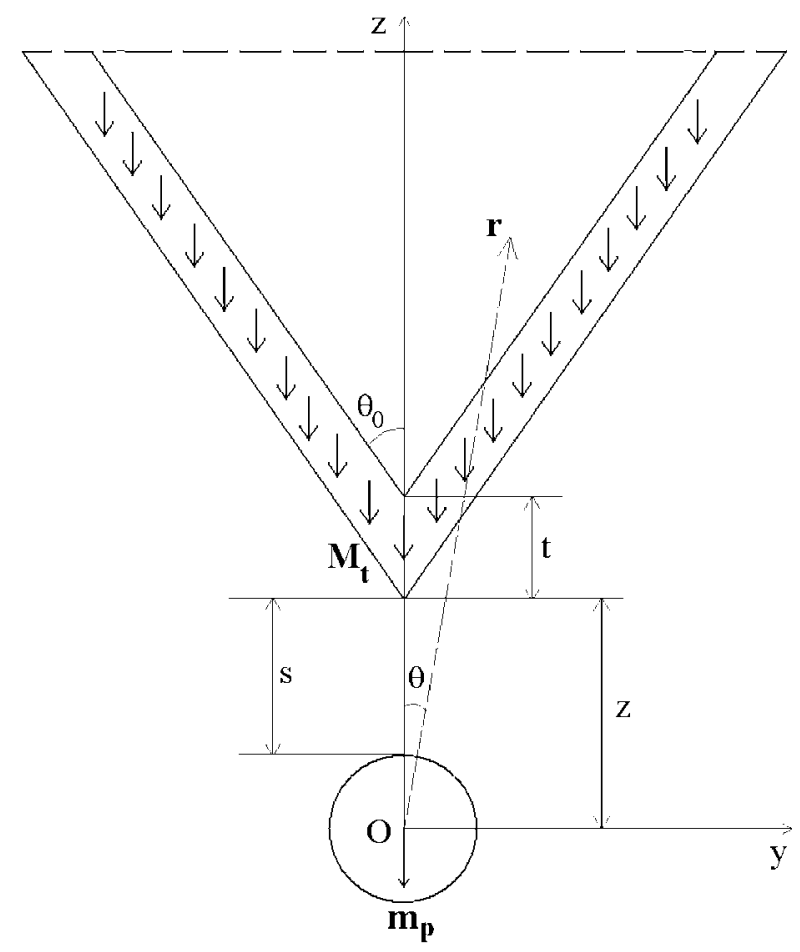

FIG. 14. Uniformly magnetized cone tip model and geometry used for calculation of the shift in the resonant frequency of the MFM cantilever.

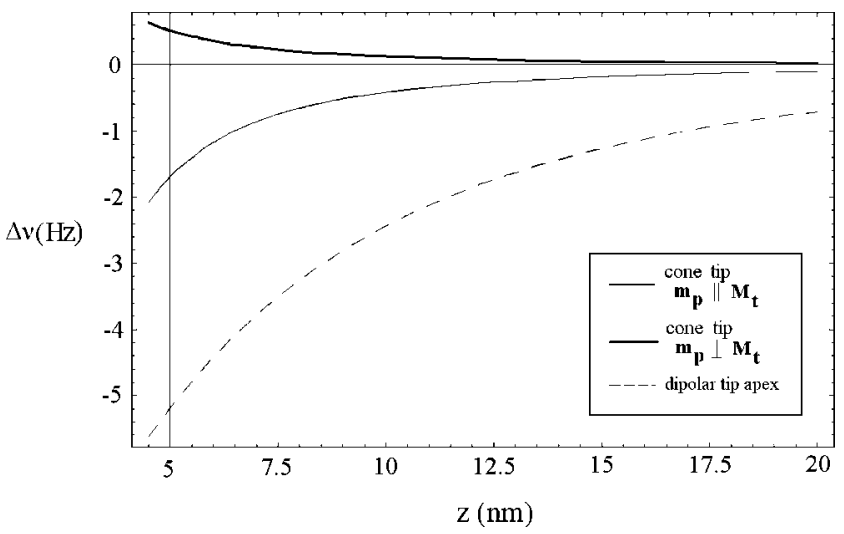

FIG. 15. Shift in the resonant frequency versus the distance between the modeled tip apex and the center of the spherical magnetite particle.

mum signal is obtained. Strictly speaking, the last assumption is valid for "ideal superparamagnetic particles" (single domain magnetic particles in which the moment rotates freely inside), and this is discussed below. First, the integral is calculated over the entire cone, so that

$$
\begin{aligned}
F_{\text {cone }}^{\prime}= & \frac{\mu_{0} m_{p} M_{t}}{4 \pi} \frac{\partial^{2}}{\partial z^{2}} \int_{0}^{\arctan \left(\frac{L \tan \theta_{0}}{z+L}\right)} \sin \theta \mathrm{d} \theta \\
& \times \int_{\frac{z \tan \theta_{0}}{\cos \theta\left(\tan \theta_{0}-\tan \theta\right)}}^{\frac{z+L}{\cos \theta}} r^{2} \mathrm{~d} r \int_{0}^{2 \pi} \mathrm{d} \phi\left(\frac{3 \cos ^{2} \theta-1}{r^{3}}\right) .
\end{aligned}
$$

$r, \theta$ and $\phi$ are the spherical coordinates and $z$ is the vertical coordinate of the apex (Fig. 14). The integral over the nonmagnetic cone core is then subtracted. The final result is rather complicated and it is presented in Fig. 15, together with that one obtained by assuming a dipole-dipole interaction between the tip apex and the particle. The results of Fig. 15 were obtained for $m_{p}=M_{d} V_{m}$, where $V_{m}$ is the magnetic volume of the particle and $M_{d}=4.46 \times 10^{5} \mathrm{~A} / \mathrm{m}$ is the magnetite domain magnetization. Strictly speaking, this is true if the thermal fluctuations of the particle magnetic moment are negligible, else, the particle moment (or magnetization) in the $\mathrm{O} z$ direction is smaller (see for example Eq. [10] below). The value $M_{d}$ can also be used if the tip field is high enough, if the temperature is low, or if the particle is magnetized downward by an external constant field. The last case will not affect the MFM image at all because the tip is influenced only by a nonuniform field. In the same figure, another case of particle-tip interaction was estimated, that one in which $\mathbf{m}_{\mathbf{p}} \perp \mathbf{M}_{\mathbf{t}}$. This could be the case of a prolate ellipsoid with pronounced anisotropy which thus lies with the long (anisotropy) axis parallel to the substrate and with its moment aligned to the direction of the anisotropy axis (the so-called "rigid dipole"). The orientation of the magnetic moment is only slightly perturbed by the tip magnetic field.

Taking into account the presence of the necessary gold layer which covers the sample,one can see that, for ideal 
superparamagnetic particles, at the lift heights attained in the experiment (corresponding to $z>10 \mathrm{~nm}$ ), the signal is close to or smaller than the lower limit of detection (around $1 \mathrm{~Hz}$ ). From the same figure, it is worth noticing that the center of an ellipsoidal rigid dipole would be viewed in an opposite magnetic contrast in comparison with a superparamagnetic particle (for the same tip position as presented in Fig. 14).

We expect from these calculations to overestimate the value of the frequency shift because in a real tip the magnetization is not uniform in the $\mathrm{O} z$ direction over the entire tip coating, and because of the thermal fluctuations of the magnetic moment inside the particle. Consequently, the real signal is smaller than that one predicted in Fig. 15 and difficult to measure with the equipment in its present form. Attempts with a more thickly coated magnetic tip were also unsuccessful, proving the overestimation of the above theoretical calculation, done for a standard tip. But further investigations are worth doing because, for example, if the particle diameter is twice as large as in our calculations, the signal will be eight times larger. Moreover, particles with higher saturation magnetization can be imaged and, finally, MFM could be performed in vacuum, which significantly increases the sensitivity of the equipment (up to one order of magnitude).

In real single domain magnetite particles, the magnetocrystalline anisotropy energy is negligible in comparison with the shape anisotropy energy (25). The latter is also small because the mean ellipticity of the real particles, according to AFM and TEM (21) determinations, is close to 1 and thus gives an account for the approximation made above. To be precise, the anisotropy energy of a real nanoparticle is negligible if $\sigma \leq 1$, where

$$
\sigma=\frac{K_{e f f} V_{m}}{k_{B} T}
$$

$K_{\text {eff }}$ is the effective anisotropy constant, $T$ is the absolute temperature, and $k_{B}$ is the Boltzmann constant. For a spherical magnetite particle this is valid up to magnetic diameters of approximately $12 \mathrm{~nm}$. In this case, $m_{p}$ is hardly influenced by the anisotropy field and only thermal fluctuations can be taken into account. Thus, we can replace the magnetization $M_{d}$ of the particle in the $\mathrm{O} z$ direction with the mean magnetization $M(H)$, calculated using the Boltzmann distribution of magnetic dipolar energies of the particle in the external field of the tip $(H)$,

$$
M(H)=M_{d} L(\xi)
$$

where $L(\xi)=\operatorname{coth} \xi-1 / \xi$ is the well-known Langevin function and $\xi=\mu_{0} m_{p} H /\left(k_{B} T\right)$ the Langevin parameter. In order to calculate the field of the tip (Fig. 16) we used the finiteelement-method-based "Magnet" program (purchased from Infolytica Ltd.). The tip was modeled as a cone with parameters determined from the SEM picture and with the hysteresis loop determined from magnetometric measurements. Another discussion is necessary here: the magnetometer measures the entire magnetic layer deposited on the cantilever and cantilever substrate, not only the magnetic volume relevant for imaging the sample. This results in overestimation of the coercive field [8]. But the coercivity does not play the main role in our problem, because the magnetite particle is superparamagnetic so that the tip influences the particle magnetization and not vice versa; consequently we may neglect this aspect for a field estimation due to the tip magnetized in the $-\mathrm{O} z$ direction. The results show that the field decreases from $3 \times 10^{5} \mathrm{~A} / \mathrm{m}$ to $10^{5} \mathrm{~A} / \mathrm{m}$ for $z \in(10-40)$ nm. For $H=10^{5} \mathrm{~A} / \mathrm{m}$, the magnetization predicted by Eq. [10] is 1.6 times smaller than $M_{d}$.

For the case of larger particles or nonspherical particles, the anisotropy field, $H_{a}=2 K_{\text {eff }} /\left(\mu_{0} M_{d}\right)$, should be also taken into account. The magnetic moment interacts in this case both with the tip field and with the anisotropy field, so that a smaller value of magnetization in the $\mathrm{O} z$ direction should be used. It depends on the exact shape and on the orientation of the anisotropy axis

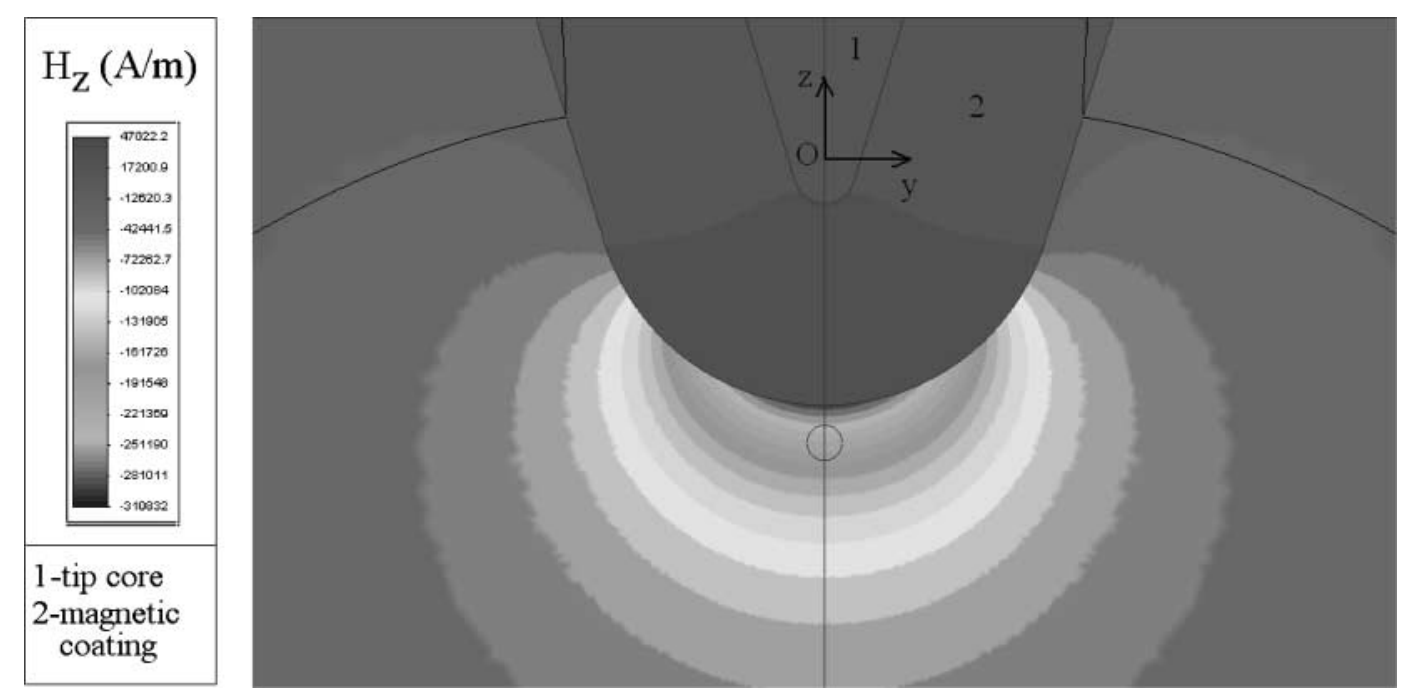

FIG. 16. Field distribution produced by an MFM tip, numerically computed with "MagNet." The closest position of a particle is also indicated. 
relative to the tip; if these are known, then the previous model can be applied but with the proper angle between $\mathbf{m}_{\mathbf{p}}$ and $\mathbf{M}_{\mathbf{t}}$ inserted. This discussion remains valid below the critical radius, above which, the particle is not single domain anymore. If the anisotropy is strong, then the rigid dipole model can be used, as already exemplified.

On the other hand, a distribution of magnetization in the tip coating was experimentally evidenced in (12) for $\mathrm{CoCr}$ coated tips of origin which was not mentioned, and presented in Fig. 17. They were used for the same microscope as in this paper. For this case, the force derivative was calculated again by integrating the dipolar field analytically over the magnetic coating (the magnetization of the particle is considered to be $M_{d}$, for comparison with the previous calculation). Reasonable assumptions about magnetization distribution for DI tips can be found in (26) but without experimental proofs. In order to have a more accurate reference for comparison, we estimated the minimium detectable force derivative, given by (27),

$$
F_{\text {min }}^{\prime}=\frac{1}{A} \sqrt{\frac{4 k k_{B} T B}{2 \pi v_{0} Q}},
$$

where $A$ is the rms amplitude of the cantilever, $Q$ is the quality factor of the resonance, and $B$ is the detection bandwidth. The rms amplitude was determined from the force calibration plot to be of the order of $10 \mathrm{~nm}$ while the quality factor of the resonance was 231. In Fig. 18, the calculated force derivatives with the last two models are presented together with the minimum detectable force derivative. One can see that the difference between the two models is not significant, and both predict force derivatives below the minimum detectable force in the region of interest for experiments $(z>10 \mathrm{~nm})$.

According to the discussions in the last two paragraphs, it is expected that magnetite particles can be imaged if operating

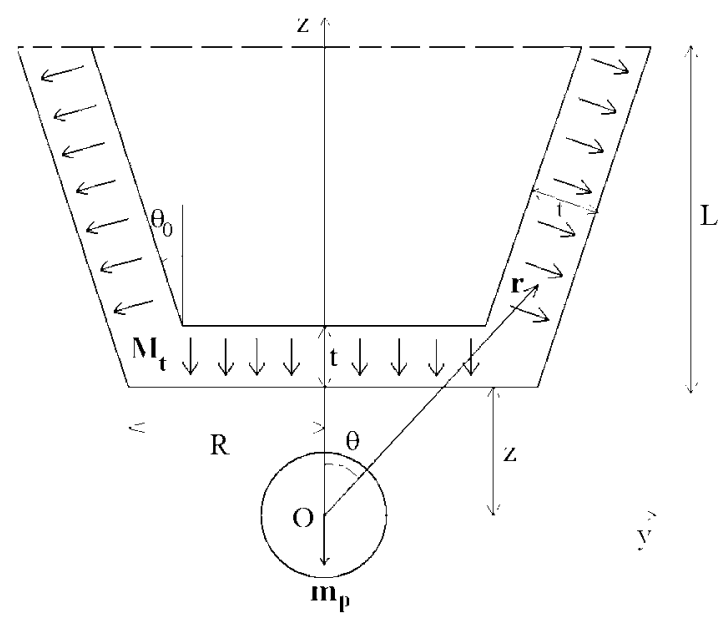

FIG. 17. Truncated cone tip model with nonuniform magnetization and geometry used for calculation of the shift in the resonant frequency of the MFM cantilever.

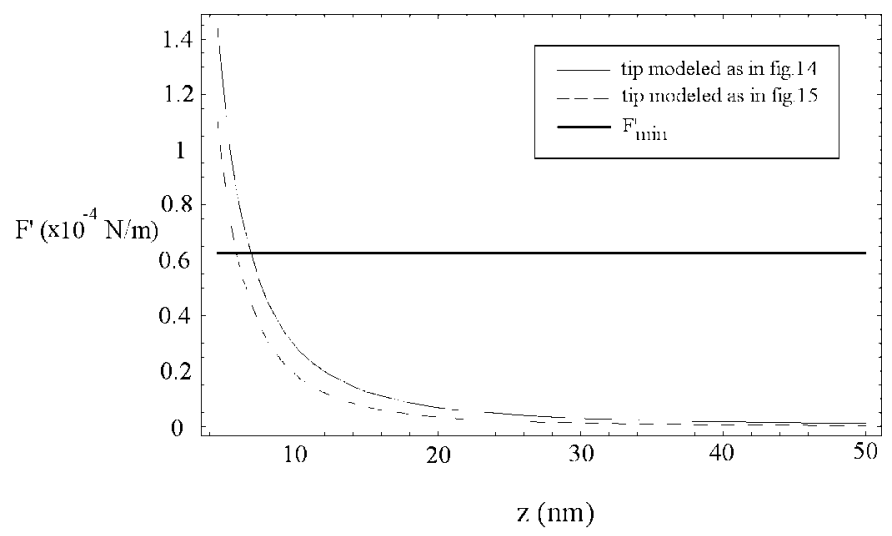

FIG. 18. Force derivative acting on the tip versus the distance between the modeled tip apex and the center of a spherical particle and the minimum detectable value of the force derivative.

in vacuum, or in air, if they are slightly larger (with diameter $\geq 15 \mathrm{~nm}$ ) than those usually dispersed in the classical magnetic fluids. The models can be used for determination of the magnetic moment of a single particle. The center of the particle can be located easily using the topographic image and the corresponding shift in the resonant frequency can be determined from the lift mode image using section analysis. After being measured at different lift heights, $m_{p}$ can be determined by fit. Alternatively, the particle height can be determined from the topographic image (and thus $m_{p}$ ), so that the mean tip magnetization in the $\mathrm{O} z$ direction can be found.

\section{CONCLUSIONS}

AFM can provide reliable height and approximate lateral diameter (after deconvolution) for most colloidal particles in this study. In the case of platelike particles the lateral diameter is also correct. In the case of latex colloids we observed the flattening of particles on the substrate, so that the measured dimensions differ from those of particles in the solvent. The height to lateral diameter ratio as well as the lateral aspect ratio were determined and compared for shape and dimensional study of all these particles. Both height and phase images are useful for characterizing the shape, hardness and dimensions of particles as well as 2D cluster composition. Second nucleation in the case of latex dispersions and surfactant in the case of Teflon-latex dispersions were observed with AFM and not with TEM. These results demonstrate that AFM is at least a versatile supplement to TEM in the characterization of colloids.

Deconvolution improves the accuracy of the measured nanoparticle diameter in some extent, but the tip apex characterization depends on the used technique. We consider that the blind characterization technique for tip reconstruction followed by deconvolution, applied on the same image to be deconvoluted, gives the best result, in good agreement with the geometrical estimation of the real particle radius and in better agreement with the TEM results than in the case of the other methods. In the case 
of larger undeformable spheres, the convolution is determined by the mean half angle of the tip and the geometrical estimation of the real diameter was successful.

The shift in the resonant frequency of an MFM probe interacting with a single-domain spherical superparamagnetic nanoparticle was estimated within the frame of several proposed models, fully discussed and compared. Our conclusion is that magnetite particles dispersed in a conventional ferrofluid produce a signal too small to be detected by the MFM technique in its present state. However, if measurements are done in vacuum, or in air but for a magnetic diameter larger than about $15 \mathrm{~nm}$, one expects to obtain an MFM image. In this case the models can be used for determining the magnetic moment of a single particle, which cannot be done with other techniques. Alternatively, the mean tip magnetization can be determined if the magnetic moment of a particle is determined after calculating its volume using the topographical image.

\section{ACKNOWLEDGMENTS}

The following "Van 't Hoff members" kindly provided the colloids: K. Butter (iron colloids), Dr. G. van Ewijk (magnetite ferrofluid M2), G. Koenderink (latex and Teflon dispersions), D. van der Beek (gibbsite particles), and D. Aarts (boehmite-silica rods). Dr. D. Bica (Romanian Academy of Sciences, Timişoara Branch) is acknowledged for providing the magnetite ferrofluid M1. We thank Dr. L. Abelmann and Eng. M. Siekman (University of Twente, MESA Research Institute) for helpful suggestions and for an attempt to image our magnetite nanoparticles with their MFM. Dr. P. Neilson (Digital Instruments), Professor E. D. Dahlberg (University of Minnesota), and Dr. B. Erné (Van 't Hoff Lab.) are acknowledged for helpful discussions and Dr. J. Wijnhoven (Van 't Hoff Lab.) for the SEM pictures of magnetic tips. This work was financially supported by the Colloid Physics Stimulation Programme initiated by Professor H. Lekkerkerker and Dr. G. van Ginkel and awarded by the University of Utrecht.

\section{REFERENCES}

1. Sarid, D., "Scanning Force Microscopy." Oxford Univ. Press, New York, 1994.

2. Grabar, K., Brown, K. R., Keating, C. D., Stranick, S. J., Tang, S., and Natan, M., Anal. Chem. 69, 471 (1997).
3. Vesenka, J., Manne, S., Giberson, R., Marsh, T., and Henderson, E., Biophys. J. 65, 992 (1993).

4. Bourgeat-Lami, E., and Lang, J., J. Colloid Interface Sci. 197, 293 (1998).

5. Porthun, S., Abelmann, L., and Lodder, C., J. Magn. Magn. Mater. 182, 238 (1998).

6. Folks, L., and Woodward, R. C., J. Magn. Magn. Mater. 190, 28 (1998).

7. Huo, S., Bishop, J. E. L., Tucker, J. W., Al-Khafaji, M. A., Rainforth, W. M., Davies, H. A., and Gibbs, M. R. J., J. Magn. Magn. Mater. 190, 17 (1998).

8. Babcock, K. L., Elings, V. B., Shi, J., Awschalom, D. D., and Dugas, M., Appl. Phys. Lett. 69(5), 705 (1996).

9. Schoenenberger, C., and Alvarado, S. F., Z. Phys. B Cond. Mat. 80, 373 (1990).

10. Vellekoop, S. J. L., Abelmann, L., Porthun, S., Lodder, J. C., and Miles, J. J., J. Magn. Magn. Mater. 193, 474 (1999).

11. Kleiber, M., Kuemmerlen, F., Lohndorf, M., Wadas, A., Weiss, D., and Wiesendanger, R., Phys. Rev. B 58, 5563 (1998).

12. Proksch, R. B., Schaffer, T. E., Moskowitz, B. M., Dahlberg, E. D., Bazylinski, D. A., and Frankel, R. B., Appl. Phys. Lett. 66(19), 2582 (1995).

13. Charles, S., and Massart, R., in "Magnetic Fluids and Applications Handbook" (B. Berkovski and V. Bashtovoi, Eds.), p. 4. Begell House, New York, 1996.

14. Pathmamanoharan, C., Zuiverloon, N. L., and Philipse, A. P., Prog. Colloids Polym. Sci. 115, 141 (2000).

15. Bica, D., Rom. Rep. Phys. 47, 3-5, 265 (1995).

16. Koenderink, G., Sacanna, S., Pathmamanoharan, C., Raşa, M., and Philipse, A., Langmuir 17(20), 6086 (2001).

17. Philipse, A. P., and Vrij, A., J. Colloid Interface Sci. 128, 121 (1989).

18. Philipse, A. P., Nechifor, A.-M., and Pathmamanoharan, C., Langmuir 10, 4451 (1994).

19. Wierenga, A. M., Lenstra, T. A. J., and Philipse, A. P., Colloids Surf. A 134, 359 (1998).

20. van der Kooij, F. M., Philipse, A. P., and Dhont, J. K. G., Langmuir 16(12), 5317 (2000).

21. Raşa, M., Eur. Phys. J. E 2(3), 265 (2000).

22. Villarrubia, J. S., J. Res. Natl. Inst. Stand. Technol. 102, 425 (1997)

23. Nioprobe Instruction Guide, Electron Microscopy Sciences, 2001.

24. Lohau, J., Kirsch, S., Carl, A., Dumpich, G., and Wassermann, E. F., J. Appl. Phys. 86(6), 3410 (1999).

25. Raikher, Yu., and Shliomis, M., Adv. Chem. Phys. 87, 595 (1994).

26. Tomlinson, S., and Hill, E. W., J. Magn. Magn. Mater. 161, 385 (1996).

27. Albrecht, T. R., Gruetter, P., Horne, D., and Rugar, D., J. Appl. Phys. 69(2), 66 (1991). 